\title{
A SEMI-IMPLICIT SCHEME FOR STATIONARY STATISTICAL PROPERTIES OF THE INFINITE PRANDTL NUMBER MODEL
}

\author{
WENFANG (WENDY) CHENG AND XIAOMING WANG* \\ FLORIDA STATE UNIVERSITY, TALLAHASSEE, FL 32306, USA
}

\begin{abstract}
We propose a semi-discrete in time semi-implicit numerical scheme for the infinite Prandtl model for convection. Besides the usual finite time convergence, this scheme enjoys the additional highly desirable feature that the stationary statistical properties of the scheme converge to those of the infinite Prandtl number model at vanishing time step. One of the key characteristics of the scheme is that it preserves the dissipativity of the infinite Prandtl number model uniformly in terms of the time step. So far as we know, this is the first rigorous result on convergence of stationary statistical properties of numerical schemes for infinite dimensional dissipative complex systems.
\end{abstract}

Key words. stationary statistical property, infinite Prandtl number model, uniformly dissipative scheme, Nusselt number

AMS subject classifications. 65M12, 65Z05, 65P99, 37M25, 76D06, 76M25, 76R10

1. Introduction. Many dynamical systems arising in applications are dissipative complex systems in the sense that they possess a compact global attractor and the dynamics on the global attractor are complex/chaotic [42]. Well-known examples include the simple Lorenz 63 model, Lorenz 96 model, the Navier-Stokes equations at large Reynolds number or Grashoff number, the Boussinesq system for convection at large Rayleigh number, the Kuramoto-Sivashinsky equation at large spatial size, and many models for the atmosphere, ocean, weather and climate etc. The dynamics of these systems are typically very complex/chaotic with generic sensitive dependence on data. Therefore, it is hardly meaningful to discuss long time behavior of a single trajectory for this kind of complex system. Instead, we should study statistical properties of the system since they are physically much more relevant than single trajectories[36, 17, 34, 32]. If the system reaches some kind of stationary state, the objects that characterize the stationary statistical properties are the invariant measures or stationary statistical solutions of the system.

With a given complex system, analytical exact expressions for statistical properties are extremely rare just as exact solution formulas are rare for single trajectory. Therefore we naturally turn to numerical methods, especially with today's powerful computers and ever advancing computational technologies. The natural question then is what kind of numerical schemes would provide good approximations for the stationary statistical properties.

In terms of trajectory approximations, we are not aware of any effective long time integrator for dissipative complex/chaotic systems in general unless the long time dynamics is trivial or the trajectory under approximation is stable [23, 19]. It is not at all clear that those numerical methods that provide efficient and accurate approximations of the continuous complex dynamical system on a finite time interval are able to provide meaningful approximation for stationary statistical properties of the system since small errors (truncation and rounding) may accumulate and grow over a long time (think about the usual error estimates with a coefficient that grows exponentially in time due to the existence of chaotic behavior/positive Lyapunov exponent). Here we forego the idea of long time fidel approximation of any single trajectory, but ask if it is possible to approximate the mean or statistical properties faithfully. The

\footnotetext{
*CORRESPONDING AUTHOR, WXM@MATH.FSU.EDU
} 
numerical study of stationary statistical properties of complex system still is a very challenging task since it involves long time integration (so that the statistical averaging is computed utilizing time averaging under the assumption of ergodicity) and computation of large number of trajectories (if no ergodicity is assumed).

We will demonstrate in this paper that a semi-discrete in time and semi-implicit scheme for the infinite Prandtl number model for convection is able to capture stationary statistical properties of the underlying infinite Prandtl number model. It seems that one of the key ingredients in the convergence of the stationary statistical properties is the uniformly dissipativity of the scheme, i.e., the scheme is dissipative uniformly with respect to the time step. Although this scheme may not approximate individual trajectory faithfully for long time due to the accumulation of truncation and rounding errors and abundant instability/chaos, we will show that stationary statistical properties characterized by the invariant measures (stationary statistical solutions) of the scheme converge to those of the continuous in time system. This gives us a strong evidence that this kind of uniformly dissipative schemes are appropriate schemes in investigating statistics.

Although the idea of uniform dissipativity and convergence of stationary statistical properties is illustrated on the infinite Prandtl number model for convection and semi-discretization in time only, we believe that the methodology works for many more complex/chaotic dynamical systems [42] and fully discretized approximations. The key ingredients are uniform (in mesh size) dissipativity and finite time uniform convergence (see [51] for a somewhat general statement). The choice of the infinite Prandtl number model is both for its physical significance (see the next section) and for the sake of simplicity in exposition.

The idea of uniformly dissipative approximation for dissipative dynamical system is a very natural one. Since the continuous in time dynamical system is dissipative (possess a global attractor), it is natural to consider numerical schemes that preserve the dissipativity in the sense that the solutions to the schemes should possess global attractors that are uniformly compact in some appropriate sense (say the union of the global attractors is pre-compact). These uniformly dissipative schemes are usually implicit in some way (to ensure long time stability) and therefore have not been very popular in practice so far. What we shall demonstrate below is that some of these uniformly dissipative schemes enjoy a highly desirable property in terms of approximating stationary statistical properties: the stationary statistical properties of the schemes converge to those of the continuous in time dynamical systems. We hope that our work will stimulate further study, both analytical and numerical, on approximating statistical properties of dissipative systems.

Earlier works on long time behavior of numerical schemes for dissipative system mainly focused on the two dimensional incompressible Navier-Stokes system and the Kuramoto-Sivashinsky equation (see $[19,25,29,39,43,15,16]$ among others) and the notion of long time stability or dissipativity. The uniform bound in the phase space and a finer/smaller space is called long time stability or dissipativity in these works (some of the authors only derived uniform bound/long time stability in the phase space without other bounds that are necessary for ensuring the uniform dissipativity of the scheme). We prefer the term uniform dissipativity since long time stability could be misleading in the sense that it may imply the scheme is global in time stable in one single phase space only which is not sufficient to ensure the existence of the global attractor. Also, none of the authors discussed stationary statistical properties of their schemes. To the best of our knowledge, our work is the first in establishing 
the convergence of stationary statistical properties and therefore the usefulness of uniformly dissipative schemes in approximating stationary statistical properties. An announcement of the main results presented here can be found [10].

The manuscript is organized as follows: we give an introduction in the first section; in section 2 we propose a semi-discrete (discrete in time) scheme for the infinite Prandtl number model and verify that it is uniformly dissipative and enjoys the property that the stationary statistical properties of the scheme converge to those of the continuous in time model; we then provide conclusion and remarks in the third section.

\section{A uniformly dissipative scheme for the infinite Prandtl number model.}

2.1. The infinite Prandtl number model for convection. One of the fundamental systems in fluid dynamics is the Boussinesq system for Raleigh-Bénard convection which is a model for convection, i.e., fluid motion induced by differential heating under Boussinesq approximation $([44,18])$. We assume that the fluids occupy the (non-dimensionalized) region $\Omega=\left[0, L_{x}\right] \times\left[0, L_{y}\right] \times[0,1]$ with periodicity imposed in the horizontal directions for simplicity.

The Boussinesq system exhibits extremely rich phenomena (see for instance [18, 44], and the recent reviews [4, 40]). In fact, the Boussinesq system is considered a fundamental paradigm for nonlinear dynamics including instabilities and bifurcations, pattern formation, chaotic dynamics and fully developed turbulence [30]. On the other hand, we have very limited mathematical knowledge on the system. Therefore various physically relevant simplifications are highly desirable in order to make progress.

For fluids such as silicone oil or the earth's mantle, the Prandtl number is large, therefore, we may formally set the Prandtl number to infinity in the non-dimensional Boussinesq system, and we arrive at the following (see for instance [4, 6, 8, 20, 44] among others) infinite Prandtl number model (non-dimensional)

$$
\begin{aligned}
\nabla p & =\Delta \mathbf{u}+R a \mathbf{k} T, \quad \nabla \cdot \mathbf{u}=0,\left.\quad \mathbf{u}\right|_{z=0,1}=0, \\
\frac{\partial T}{\partial t}+\mathbf{u} \cdot \nabla T & =\Delta T,\left.\quad T\right|_{z=0}=1,\left.T\right|_{z=1}=0
\end{aligned}
$$

where $\mathbf{u}$ is the Eulerian velocity of the fluid, $p$ represents the kinematic pressure of the fluid, $T$ is the temperature of the fluid, $\mathbf{k}$ is a unit vector in the $z$ direction, and $R a$ is the Rayleigh number measuring the ratio of differential heating over overall dissipation.

It is well-known that for complex systems such as convection system at large Rayleigh number where turbulent/chaotic behavior abound (see for instance $[6,18,30$, $4,40]$ ), statistical properties for such systems are much more important and physically relevant than single trajectories $[36,17,32,34]$.

Although there have been extensive works on heat transport in Rayleigh-Bénard convection $[1,4,6,7,20,40,26,27]$, basic statistical properties of the system such as the heat transport in the vertical direction quantified by the Nusselt number and the mean velocity field are not very well understood. On the other hand, the infinite Prandtl number model is much simpler than the Boussinesq system since the NavierStokes equations are replaced by the Stokes equations (and therefore the phase space is that of the temperature only). We also know that the statistics of the infinite Prandtl number model are close to those of the Boussinesq system at large Prandtl number $[47,48,49]$. Therefore it makes sense for us to study some fundamental statistical properties of convection utilizing the simple infinite Prandtl number model since we 
can generally expect to push to physically more interesting higher Rayleigh number without sacrificing accuracy with the currently available computing resource.

In our case of infinite Prandtl number convection at large Rayleigh number, even the computation on order one time scale (diffusive time scale) is a challenge since it in fact is a long time integration in disguise. To see this, we can rewrite the infinite Prandtl number model as

$$
\frac{\partial T}{\partial t}+R a A^{-1}(\mathbf{k} T) \cdot \nabla T=\Delta T,\left.\quad T\right|_{z=0}=1,\left.T\right|_{z=1}=0
$$

where $A$ denotes the Stokes operator with viscosity one and the associated boundary conditions. It is then apparent that this is an advection dominated problem (large Péclet number) for large Rayleigh number $R a$. We divide both sides of the equation by $R a$ and introduce the fast time scale $\tau=R a t$ we may rewrite the infinite Prandtl number model in the following alternative form with an order one advection term

$$
\frac{\partial T}{\partial \tau}+A^{-1}(\mathbf{k} T) \cdot \nabla T=\frac{1}{R a} \Delta T .
$$

It appears that the leading order dynamics at large Rayleigh number is the non-local advection equation $\frac{\partial T}{\partial \tau}+A^{-1}(\mathbf{k} T) \cdot \nabla T=0$. However, this is only valid on order one time scale for the fast time $\tau$. What we are interested in is order one time scale for the diffusive time $t$ which means long time for the fast time $\tau$ (of the order of $R a$ ).

2.2. A semi-discrete in time scheme. In this subsection, we provide a specific semi-discrete in time convergent dissipative scheme for the infinite Prandtl number model. The scheme is semi-implicit and utilizes a background temperature profile. Indeed, consider a generic background temperature profile $\tau(z)$ which satisfies the non-homogeneous Dirichlet boundary condition of $T$. We introduce the perturbative temperature field $\theta=T-\tau$. The exact form of the background profile $\tau$ to be used will be specified below. It is easy to see that $\theta$ satisfies the following equation

$$
\frac{\partial \theta}{\partial t}+R a A^{-1}(\mathbf{k} \theta) \cdot \nabla \theta+R a A^{-1}(\mathbf{k} \theta)_{3} \tau^{\prime}(z)=\Delta \theta+\tau^{\prime \prime}(z),\left.\quad \theta\right|_{z=0,1}=0,
$$

and we are searching for solution in the space $H_{0, p e r}^{1}$ (the subspace of $H^{1}$ with zero trace in the $z$ direction and periodic in the horizontal directions). Here $A^{-1}(\mathbf{k} \theta)_{3}$ represents the third component (vertical velocity) of $A^{-1}(\mathbf{k} \theta)$.

The semi-implicit semi-discrete in time scheme that we propose is given by,

$$
(2.6) \frac{\theta^{n+1}-\theta^{n}}{k}+R a A^{-1}\left(\mathbf{k} \theta^{n}\right) \cdot \nabla \theta^{n+1}+R a A^{-1}\left(\mathbf{k} \theta^{n+1}\right)_{3} \tau^{\prime}(z)=\Delta \theta^{n+1}+\tau^{\prime \prime}(z)
$$

where $\theta^{n}$ denotes the approximate solution at time $k n$ where $k$ is the time step. A more accurate notation would be $\theta_{k}^{n}$ to indicate the dependence on the time step $k$. However, we will suppress the $k$ dependence in the notion for simplicity except in the convergence proof.

Notice that the scheme is linear although the PDE is nonlinear.

Also, we would arrive at a different scheme if we discretize in time first and then apply the translation/background profile (see (3.2), for the case of $\lambda=0$ ).

Following the pioneer works of Constantin and Doering [7, 8], we set background temperature profile $\tau$ to be a locally smoothed (mollified) version of the following 
piecewise linear function

$$
\tau(z)=\left\{\begin{array}{cc}
1-\frac{z}{2 \delta}, & 0 \leq z \leq \delta \\
\frac{1}{2}, & \delta \leq z \leq 1-\delta \\
\frac{1-z}{2 \delta} & 1-\delta \leq z \leq 1
\end{array}\right.
$$

The choice of the parameter $\delta$ will be specified later.

We would like to remark here that the typical choice of $\tau$ being the conduction state $1-z$ is not a good one. In fact, the linearized equation is unstable in this case [6] and the solutions (to the linearized problem) grow without bound for generic initial data. Therefore we have to utilize the non-linear term (this is where the new background profile comes into picture) to stabilize the whole system. It is also worthwhile to point out that boundary conditions play an important role in the stabilization process. For instance if we choose $\tau=1-z$ (the pure conduction state) and utilize periodicity for the perturbative variables in all three directions (the so-called homogeneous Rayleigh-Bénard convection), the nonlinear system is not stable [5] (look at solutions that are $z$ independent).

2.3. Well-posedness. The well-posedness of the discrete scheme follows from Lax-Milgram theorem [33].

The weak formulation of the scheme can be derived by multiplying the scheme (2.6) by a test a test function $\psi \in H_{0, p e r}^{1}$ and integrating by parts. The weak formulation of the discrete scheme can be re-written into the form

$$
B_{n}\left(\theta^{n+1}, \psi\right)=L_{n}(\psi)
$$

where

$$
\begin{aligned}
& B_{n}\left(\theta^{n+1}, \psi\right) \\
= & \left(\frac{1}{k} \theta^{n+1}+R a A^{-1}\left(\mathbf{k} \theta^{n}\right) \cdot \nabla \theta^{n+1}+R a A^{-1}\left(\mathbf{k} \theta^{n+1}\right)_{3} \tau^{\prime}, \psi\right)+\left(\nabla \theta^{n+1}, \nabla \psi\right)
\end{aligned}
$$

$$
=-\left(\tau^{\prime}(z), \frac{\partial \psi}{\partial z}\right)+\left(\frac{1}{k} \theta^{n}, \psi\right)
$$

It is easy to see that $B_{n}$ is a continuous bilinear form on $H_{0, p e r}^{1} \times H_{0, p e r}^{1}$ and $L_{n}$ is a continuous linear functional on $H_{0, p e r}^{1}$. We only need to verify the coercivity for $B_{n}$ in order to show the solvability thanks to Lax-Milgram theorem.

For this purpose we notice that, thanks to the specific form of the background profile $\tau$ given in (2.7), the homogeneous boundary conditions for $\theta^{n+1},\left(A^{-1}\left(\mathbf{k} \theta^{n+1}\right)\right)_{3}$, $\nabla\left(A^{-1}\left(\mathbf{k} \theta^{n+1}\right)\right)_{3}$, elliptic regularity (for the Stokes operator) and Poincaré inequality (three times), there exists a constant $c_{1}$ independent of $k, n, R a$, such that,

$$
R a\left|\int_{\Omega} \tau^{\prime}(z)\left(A^{-1}\left(\mathbf{k} \theta^{n+1}\right)\right)_{3} \theta^{n+1}\right| \leq c_{1} \delta^{2} R a\left\|\nabla \theta^{n+1}\right\|^{2} \leq \frac{1}{4}\left\|\nabla \theta^{n+1}\right\|^{2}
$$

provided that we choose ${ }^{1}$

$$
\delta=\left(4 c_{1} R a\right)^{-\frac{1}{2}}
$$

\footnotetext{
${ }^{1}$ The choice of $\tau$ or $\delta$ given here is not optimal. A near optimal choice would be $\delta \sim R a^{-\frac{1}{3}}$ but the control on the linear destabilizing term is much longer $[8,12,49]$. We use the simple one since optimal bound is not our goal here.
} 
Therefore

$$
B_{n}\left(\theta^{n+1}, \theta^{n+1}\right) \geq \frac{1}{k}\left\|\theta^{n+1}\right\|^{2}+\frac{3}{4}\left\|\nabla \theta^{n+1}\right\|^{2}
$$

which proves the coercivity. Here and elsewhere $\|\theta\|=\sqrt{\int_{\Omega}|\theta|^{2}}$ denotes the spatial $L^{2}$ norm of $\theta$, and $\|\theta\|_{\infty}=\operatorname{esssup}_{\Omega}|\theta|$ denotes the spatial $L^{\infty}$ norm of $\theta$.

This ends the proof of the well-posedness of the discrete scheme.

2.4. Uniform dissipativity. Next, we prove the uniform dissipativity. Here and below, the $c_{j}$ s denote generic constants independent of $k, n$ (but which may depend on the Rayleigh number).

We first derive a uniform bound in the $L^{2}$ space. For this purpose we take the inner product of the scheme with $\psi=\theta^{n+1}$ and utilize the identity $(a-b, a)=$ $\frac{1}{2}\left(|a|^{2}-|b|^{2}+|a-b|^{2}\right)$ together with the estimate on the destabilizing term (2.11), and we have

$$
\begin{aligned}
& \frac{1}{2 k}\left(\left\|\theta^{n+1}\right\|^{2}-\left\|\theta^{n}\right\|^{2}+\left\|\theta^{n+1}-\theta^{n}\right\|^{2}\right)+\left\|\nabla \theta^{n+1}\right\|^{2} \\
\leq & \left\|\tau^{\prime}\right\|\left\|\nabla \theta^{n+1}\right\|+R a\left|\int_{\Omega} \tau^{\prime}(z)\left(A^{-1}\left(\mathbf{k} \theta^{n+1}\right)\right)_{3} \theta^{n+1}\right| \\
\leq & \left\|\tau^{\prime}\right\|^{2}+\frac{1}{2}\left\|\nabla \theta^{n+1}\right\|^{2} .
\end{aligned}
$$

Therefore, there exists a constant $c_{2}$, such that

$$
\frac{1}{k}\left(\left\|\theta^{n+1}\right\|^{2}-\left\|\theta^{n}\right\|^{2}+\left\|\theta^{n+1}-\theta^{n}\right\|^{2}\right)+\left\|\nabla \theta^{n+1}\right\|^{2} \leq 2\left\|\tau^{\prime}\right\|^{2} \leq c_{2} R a^{\frac{1}{2}}
$$

which further implies, thanks to Poincaré inequality,

$$
(1+k)\left\|\theta^{n+1}\right\|^{2} \leq\left\|\theta^{n}\right\|^{2}+c_{2} k R a^{\frac{1}{2}} .
$$

This leads to, with the help of a simple iteration,

$$
\left\|\theta^{n+1}\right\|^{2} \leq(1+k)^{-(n+1)}\left\|\theta_{0}\right\|^{2}+c_{2} R a^{\frac{1}{2}} .
$$

This is a uniform estimate in the $L^{2}$ space.

A byproduct of this estimate is that

$$
\frac{1}{N} \sum_{n=0}^{N}\left\|\nabla \theta^{n+1}\right\|^{2} \leq \frac{\left\|\theta_{0}\right\|^{2}}{k N}+c_{2} R a^{\frac{1}{2}}
$$

which is a bound on the Nusselt number in this discretized case for large $N$ (see the definition later for Nusselt number $(2.58,2.59)$ ), and a bound in $L^{2}\left(H^{1}\right)$ for the scheme.

We also have

$$
\sum_{n=0}^{N}\left\|\theta^{n+1}-\theta^{n}\right\|^{2} \leq\left\|\theta_{0}\right\|^{2}+c_{2} k N R a^{\frac{1}{2}}
$$


In order to obtain uniform estimates in $H^{1}$, we take the inner product of the scheme with $\psi=-\Delta \theta^{n+1}$ and we have

$$
\begin{aligned}
& \frac{1}{2 k}\left(\left\|\nabla \theta^{n+1}\right\|^{2}-\left\|\nabla \theta^{n}\right\|^{2}+\left\|\nabla\left(\theta^{n+1}-\theta^{n}\right)\right\|^{2}\right)+\left\|\Delta \theta^{n+1}\right\|^{2} \\
\leq & \left\|\tau^{\prime \prime}\right\|\left\|\Delta \theta^{n+1}\right\|+R a\left\|\tau^{\prime}\right\|\left\|A^{-1}\left(\mathbf{k} \theta^{n+1}\right)\right\|_{\infty}\left\|\Delta \theta^{n+1}\right\|+R a\left\|A^{-1}\left(\mathbf{k} \theta^{n}\right)\right\|_{\infty}\left\|\nabla \theta^{n+1}\right\|\left\|\Delta \theta^{n+1}\right\| \\
\leq & \left\|\tau^{\prime \prime}\right\|\left\|\Delta \theta^{n+1}\right\|+c_{3} R a\left\|\tau^{\prime}\right\|\left\|\theta^{n+1}\right\|\left\|\Delta \theta^{n+1}\right\|+c_{4} R a\left\|\theta^{n}\right\|\left\|\theta^{n+1}\right\|^{\frac{1}{2}}\left\|\Delta \theta^{n+1}\right\|^{\frac{3}{2}} \\
\leq & \frac{1}{2}\left\|\Delta \theta^{n+1}\right\|^{2}+c_{5}
\end{aligned}
$$

where we have applied the regularity result for the Stokes operator, the Sobolev imbedding of $H^{2}$ into $L^{\infty}$, interpolation inequality, the uniform $L^{2}$ estimate $(2.16)$ and Hölder type inequality.

This implies

$$
(1+k)\left\|\nabla \theta^{n+1}\right\|^{2} \leq\left\|\nabla \theta^{n}\right\|^{2}+2 c_{6} k
$$

which further implies, with the help of a simple iteration,

$$
\left\|\nabla \theta^{n+1}\right\|^{2} \leq(1+k)^{-n}\left\|\nabla \theta^{1}\right\|^{2}+2 c_{6} .
$$

This is the desired uniform estimates in the $H^{1}$ space, i.e., there is a uniform in $k$ bounded absorbing ball in $H^{1}$ which attracts all solutions with $L^{2}$ initial data.

Uniform estimates in Sobolev spaces with more derivatives can be derived just as in the case of continuous in time system. Here we demonstrate that the $H^{2}$ norm of the solution is asymptotically uniformly bounded in time, i.e., there is a absorbing ball in $H^{2}$ which attracts all solutions with $L^{2}$ initial data uniformly for all $k$.

For this purpose we apply $\Delta$ to both sides of the scheme (2.6) and then multiply the scheme by $\Delta \theta^{n+1}$ and integrate over the domain. This leads to the following

$$
\begin{aligned}
& \frac{1}{2 k}\left(\left\|\Delta \theta^{n+1}\right\|^{2}-\left\|\Delta \theta^{n}\right\|^{2}+\left\|\Delta\left(\theta^{n+1}-\theta^{n}\right)\right\|^{2}\right)+\left\|\nabla \Delta \theta^{n+1}\right\|^{2} \\
\leq & \left\|\tau^{(4)}\right\|\left\|\Delta \theta^{n+1}\right\|+R a\left(\left\|\Delta\left(A^{-1}\left(\mathbf{k} \theta^{n}\right)\right)\right\|_{L^{6}}\left\|\nabla \theta^{n+1}\right\|_{L^{3}}+2\left\|\nabla A^{-1}\left(\mathbf{k} \theta^{n}\right)\right\|_{L^{\infty}}\left\|\nabla^{2} \theta^{n+1}\right\|\right)\left\|\Delta \theta^{n+1}\right\| \\
& +R a\left(\left\|\Delta\left(A^{-1}\left(\mathbf{k} \theta^{n+1}\right)\right)\right\|\left\|\tau^{\prime}\right\|_{L^{\infty}}+2\left\|\nabla\left(A^{-1}\left(\mathbf{k} \theta^{n+1}\right)\right)\right\|\left\|\nabla \tau^{\prime}\right\|_{L^{\infty}}+\left\|A^{-1}\left(\mathbf{k} \theta^{n+1}\right)\right\|\left\|\Delta \tau^{\prime}\right\|_{L^{\infty}}\right)\left\|\Delta \theta^{n+1}\right\| \\
\leq & c_{7}\left(\left\|\Delta \theta^{n+1}\right\|+\left\|\Delta \theta^{n+1}\right\|^{2}\right) \\
\leq & c_{8}\left(\left\|\Delta \theta^{n+1}\right\|+\left\|\nabla \Delta \theta^{n+1}\right\|\left\|\nabla \theta^{n+1}\right\|\right) \\
\leq & \frac{1}{2}\left\|\nabla \Delta \theta^{n+1}\right\|^{2}+c_{9}
\end{aligned}
$$

where we have applied the identify $\int A^{-1}\left(\mathbf{k} \theta^{n}\right) \nabla \Delta \theta^{n+1} \Delta \theta^{n+1}=0$, Hölder's inequality, elliptic regularity, Sobolev imbedding, Cauchy-Schwarz, and interpolation inequality.

This leads to the inequality

$$
(1+k)\left\|\Delta \theta^{n+1}\right\|^{2} \leq\left\|\Delta \theta^{n}\right\|^{2}+2 c_{9} k
$$

which further implies, with the help of a simple iteration,

$$
\left\|\Delta \theta^{n+1}\right\|^{2} \leq(1+k)^{-n+1}\left\|\Delta \theta^{2}\right\|^{2}+2 c_{9} .
$$

This is the desired uniform estimates in the $H^{2}$ space, i.e., there is a uniform in $k$ bounded absorbing ball in $H^{2}$ which attracts all solutions with $L^{2}$ initial data. 
To summarize, we have the following lemma

LEMma 1 (Uniform bound/dissipativity). There exists a constant $c_{9}$ independent of the time step $k$ such that the scheme (2.6) possess an absorbing ball in $H^{1}$ and $H^{2}$ with radius $2 \sqrt{c_{9}}$ which attracts all bounded sets in $L^{2}$.

2.5. Consistency and convergence. Here we check the consistency first since this is what we need in the sequel.

Multiplying the scheme (2.6) by $k\left(\theta^{n+1}-\theta^{n}\right)$ and integrating over the domain we have

$$
\begin{aligned}
\left\|\theta^{n+1}-\theta^{n}\right\|^{2} \leq & k\left\{-\frac{1}{2}\left(\left\|\nabla \theta^{n+1}\right\|^{2}-\left\|\nabla \theta^{n}\right\|^{2}+\left\|\nabla\left(\theta^{n+1}-\theta^{n}\right)\right\|^{2}\right)\right. \\
& \left.+\left\|\tau^{\prime}\right\|\left\|\nabla\left(\theta^{n+1}-\theta^{n}\right)\right\|+c_{10}\left\|\theta^{n}\right\|_{\infty}\left\|\nabla \theta^{n+1}\right\|\left\|\theta^{n+1}-\theta^{n}\right\|+c_{11}\left\|\theta^{n+1}\right\|_{\infty}\left\|\tau^{\prime}\right\|\left\|\theta^{n+1}-\theta^{n}\right\|\right\} \\
\leq & k\left(c_{12}+c_{13}\left\|\theta^{n+1}-\theta^{n}\right\|\right)
\end{aligned}
$$

where we have applied Cauchy-Schwarz inequality, Hölder's inequality, elliptic regularity and uniform bounds in $H^{1}(2.20)$.

This implies the following consistency result

$$
\left\|\theta^{n+1}-\theta^{n}\right\| \leq c_{14} k^{\frac{1}{2}} .
$$

provided that $\theta_{0} \in H_{0, p e r}^{1}$.

If we assume $\theta_{0} \in H_{0, p e r}^{1} \bigcap H^{2}$, we may deduce from the scheme (2.6) and the uniform bound (2.22) the following stronger consistency result

$$
\left\|\theta^{n+1}-\theta^{n}\right\| \leq c_{15} k .
$$

Next, we show that the solutions to the scheme converge to the solution of the infinite Prandtl number model in $L^{2}\left(0, T^{*}, L^{2}\right)$ for any given time $T^{*}>0$ as $k \rightarrow 0$.

For this purpose, we rewrite the scheme (2.6) as

$$
\begin{aligned}
& \frac{\partial \tilde{\theta}_{k}(t)}{\partial t}+R a A^{-1}\left(\mathbf{k} \theta_{k}(t)\right) \cdot \nabla \theta_{k}(t+k)+R a A^{-1}\left(\mathbf{k} \theta_{k}(t+k)\right)_{3} \tau^{\prime}(z) \\
= & \Delta \theta_{k}(t+k)+\tau^{\prime \prime}(z)
\end{aligned}
$$

where

$$
\begin{aligned}
& \theta_{k}(t)=\theta_{k}^{n}, t \in[n k,(n+1) k), \\
& \tilde{\theta}_{k}(t)=\theta_{k}^{n}+\frac{t-n k}{k}\left(\theta_{k}^{n+1}-\theta_{k}^{n}\right), t \in[n k,(n+1) k) .
\end{aligned}
$$

The estimates $(2.16,2.17)$ imply that $\theta_{k}$ and $\tilde{\theta}_{k}$ are uniformly (in $k$ ) bounded in $L^{\infty}\left(0, T^{*} ; L^{2}\right)$ and $L^{2}\left(0, T^{*} ; H_{0, p e r}^{1}\right)$. Hence we have a subsequence, still denoted $\theta_{k}$ and $\tilde{\theta}_{k}$ and $\theta, \tilde{\theta} \in L^{\infty}\left(0, T^{*} ; L^{2}\right) \bigcap L^{2}\left(0, T^{*} ; H_{0, p e r}^{1}\right)$ such that

$$
\begin{aligned}
& \theta_{k} \rightarrow \theta, \text { weak } * \text { in } L^{\infty}\left(0, T^{*} ; L^{2}\right), \\
& \theta_{k} \rightarrow \theta, \text { weak in } L^{2}\left(0, T^{*} ; H_{0, p e r}^{1}\right), \\
& \tilde{\theta}_{k} \rightarrow \tilde{\theta}, \text { weak } * \operatorname{in} L^{\infty}\left(0, T^{*} ; L^{2}\right), \\
& \tilde{\theta}_{k} \rightarrow \tilde{\theta}, \text { weak in } L^{2}\left(0, T^{*} ; H_{0, p e r}^{1}\right) .
\end{aligned}
$$


It is also easy to check, thanks to (2.18), for any $a<T^{*}$,

$$
\begin{array}{r}
\int_{0}^{T^{*}-a}\left\|\theta_{k}(t+k)-\theta_{k}(t)\right\|^{2} d t \leq c_{16} k, \\
\int_{0}^{T^{*}}\left\|\theta_{k}(t)-\tilde{\theta}_{k}(t)\right\|^{2} d t \leq c_{17} k .
\end{array}
$$

Therefore

$$
\begin{aligned}
\theta & =\tilde{\theta}, \\
\theta_{k}(\cdot+k) & \rightarrow \theta, \text { weak } * \text { in } L^{\infty}\left(0, T^{*} ; L^{2}\right), \\
\theta_{k}(\cdot+k) & \rightarrow \theta, \text { weak in } L^{2}\left(0, T^{*} ; H_{0, p e r}^{1}\right)
\end{aligned}
$$

Further more, thanks to a compactness theorem due to Témam ([41] Ch. 13, Theorem 13.3, which states that a bounded set $\mathcal{G} \subset L^{1}\left(0, T^{*} ; Y\right) \cap L^{p}\left(0, T^{*} ; X\right), p>1$ with $X, Y$ being two Banach spaces and the injection of $Y$ into $X$ being compact, and $\sup _{g \in \mathcal{G}} \int_{0}^{T^{*}-a}\|g(a+s)-g(s)\|_{X}^{p} d s \rightarrow 0$, as $a \rightarrow 0$, is necessarily pre-compact in $\left.L^{q}\left(0, T^{*} ; X\right), \forall q \in[1, p)\right)$, there exists a sub-subsequence of $\tilde{\theta}_{k}$ which convergence strongly in $L^{q}\left(0, T^{*} ; L^{2}\right), \forall q \in[1, p)$. Indeed, testing the scheme (2.25) against a test function $v$ and integrating from $t$ to $t+a$, we have

$$
\begin{aligned}
& (2.37)\left(\tilde{\theta}_{k}(t+a)-\tilde{\theta}_{k}(t), v\right) \mid \\
& \leq \int_{t}^{t+a}\left\{\left\|\nabla \theta_{k}(s+k)\right\|\|\nabla v\|+\left\|\tau^{\prime}\right\|\left\|\frac{\partial v}{\partial z}\right\|+R a\left\|A^{-1}\left(\mathbf{k} \theta_{k}(s)\right)\right\|_{L^{\infty}}\left\|\theta_{k}(s+k)\right\|\|\nabla v\|\right. \\
& \left.\quad+R a\left\|A^{-1}\left(\mathbf{k} \theta_{k}(s+k)\right)_{3}\right\|_{L^{\infty}}\left\|\tau^{\prime}\right\|\|v\|\right\} d s \\
& \leq c_{18}\|\nabla v\| a^{\frac{1}{2}}
\end{aligned}
$$

where we have applied the regularity for the Stokes operator, Sobolev imbedding, Poincaré inequality, and the a priori estimates in $L^{\infty}\left(L^{2}\right)$ and $L^{2}\left(H^{1}\right)$ valid for $L^{2}$ initial data. Now set $v=\tilde{\theta}_{k}(t+a)-\tilde{\theta}_{k}(t)$ and utilize the $L^{2}\left(H^{1}\right)$ estimate on $\tilde{\theta}_{k}$, we have

$$
\int_{0}^{T^{*}-a}\left\|\tilde{\theta}_{k}(t+a)-\tilde{\theta}_{k}(t)\right\|^{2} \leq c_{19} a^{\frac{1}{2}} .
$$

This implies the strong convergence by Témam's compactness theorem.

Combining this strong convergence in $L^{q}\left(L^{2}\right), q \in[1,2)$ with the uniform $L^{\infty}\left(0, T^{*} ; L^{2}\right)$ estimate, we conclude that the sub-subsequence in fact converges strongly in $L^{q}\left(0, T^{*} ; L^{2}\right), \forall q \in$ $[1, \infty)$. Hence we may summarize the a priori estimates as

$$
\begin{aligned}
& (2.39) \quad \theta_{k}(\cdot), \theta_{k}(\cdot+k), \tilde{\theta}_{k}(\cdot), \tilde{\theta}_{k}(\cdot+k) \rightarrow \theta(\cdot), \text { in } L^{q}\left(0, T^{*} ; L^{2}\right), \forall q \in[1, \infty), \\
& (2.40) \quad \theta_{k}(\cdot), \theta_{k}(\cdot+k), \tilde{\theta}_{k}(\cdot), \tilde{\theta}_{k}(\cdot+k) \rightarrow \theta(\cdot), \text { weakly in } L^{2}\left(0, T^{*} ; H_{0, p e r}^{1}\right) .
\end{aligned}
$$

Now for any $\phi \in H_{0, \text { per }}^{1}$ and $\psi \in C^{1}\left(\left[0, T^{*}\right]\right)$ with $\psi\left(T^{*}\right)=0$, we can rewrite the scheme (2.25) in the following weak form

$$
\begin{aligned}
& \int_{0}^{T^{*}} \int_{\Omega}\left\{-\tilde{\theta}_{k}(\mathbf{x}, t) \phi(\mathbf{x}) \psi^{\prime}(t)+R a A^{-1}\left(\mathbf{k} \theta_{k}(\mathbf{x}, t)\right) \cdot \nabla \theta_{k}(\mathbf{x}, t+k) \phi(\mathbf{x}) \psi(t)\right. \\
& +R a A^{-1}\left(\mathbf{k} \theta_{k}(\mathbf{x}, t+k)\right)_{3} \tau^{\prime}(z) \phi(\mathbf{x}) \psi(t)+\nabla \theta_{k}(\mathbf{x}, t+k) \cdot \nabla \phi(\mathbf{x}) \psi(t) \\
& \left.+\tau^{\prime}(z) \frac{\partial}{\partial z} \phi(\mathbf{x}) \psi(t)\right\} d \mathbf{x} d t \\
& =\int_{\Omega} \theta_{0}(\mathbf{x}) \phi(\mathbf{x}) \psi(0) d \mathbf{x} .
\end{aligned}
$$


Utilizing the strong $L^{q}\left(L^{2}\right)$ convergence (2.39) and the weak $L^{2}\left(H_{0, p e r}^{1}\right)$ convergence (2.40) together with elliptic regularity, we can pass to the limit as $k \rightarrow 0$ and we arrive at

$\int_{0}^{T^{*}} \int_{\Omega}\left\{-\theta \phi \psi^{\prime}+R a A^{-1}(\mathbf{k} \theta) \cdot \nabla \theta \phi \psi+R a A^{-1}(\mathbf{k} \theta)_{3} \tau^{\prime} \phi \psi+\nabla \theta \cdot \nabla \phi \psi+\tau^{\prime} \frac{\partial}{\partial z} \phi \psi\right\} d \mathbf{x} d t=\int_{\Omega} \theta_{0} \phi \psi(0)$ $(2.42)$

which is exactly the weak form of the infinite Prandtl number model. Since the infinite Prandtl number model possesses a unique solution, $\theta$ must be the unique solution and hence the whole sequence of $\theta_{k}$ and $\tilde{\theta}_{k}$ converges to $\theta$ as any subsequence has a sub-subsequence that converges to the same limit $\theta$.

We summarize the result here as

Lemma 2 (Consistency and convergence). For any given $T^{*}>0$ and $\theta_{0} \in L^{2}$, the solution to the numerical scheme (2.25) converges to the solution of the infinite Prandtl number model, i.e.,

$$
\begin{aligned}
& \theta_{k}(\cdot), \theta_{k}(\cdot+k), \tilde{\theta}_{k}(\cdot), \tilde{\theta}_{k}(\cdot+k) \rightarrow \theta(\cdot), \text { in } L^{q}\left(0, T^{*} ; L^{2}\right), \forall q \in[1, \infty), \\
& \theta_{k}(\cdot), \theta_{k}(\cdot+k), \tilde{\theta}_{k}(\cdot), \tilde{\theta}_{k}(\cdot+k) \rightarrow \theta(\cdot), \text { weakly in } L^{2}\left(0, T^{*} ; H_{0, p e r}^{1}\right)
\end{aligned}
$$

where $\theta$ is the unique solution to the infinite Prandtl number model.

Moreover, if $\theta_{0} \in H_{0, p e r}^{1} \bigcap H^{2}$, then there exists a generic constant $c_{15}$ independent of $k, n$ such that

$$
\left\|\theta^{n+1}-\theta^{n}\right\| \leq c_{15} k
$$

We have established $L^{q}\left(L^{2}\right), \forall q<\infty$ convergence of the numerical scheme. Uniform in time (on finite time interval, i.e. $L^{\infty}\left(0, T^{*} ; L^{2}\right)$ ) of the scheme can be established as well if we assume all the compatibility conditions needed (so that the exact solution is smooth enough up to the initial time $t=0$, see [41] for the case of Navier-Stokes equations). If no high order compatibility condition is assumed, one can show the uniform in time convergence on any finite interval modulus an initial layer (see [22] for the case of Navier-Stokes equations). Uniform convergence without enough compatibility conditions is needed for the proof of convergence of the global attractors [51] but not required here and hence we skip the details.

2.6. Convergence of the stationary statistical properties. As we mentioned earlier, for complex systems with chaotic/turbulent behavior, statistical properties are much more important than individual trajectories. In fact it is essentially hopeless to try to find approximation schemes that possess the property that the approximate trajectory remain close to the "true" trajectory for all time due to abundant sensitive dependence on data and positive Lyapunov exponents. ${ }^{2}$ Therefore, the natural question to ask is if stationary statistical properties are well approximated. These stationary statistical properties are characterized by stationary statistical solutions or invariant measures of the system. Hence the question that we ask here is if the invariant measures of the discrete time approximation approximate the invariant measures of the continuous in time infinite Prandtl number model.

\footnotetext{
${ }^{2}$ There is a notable exception when the system possesses explicit hyperbolic structure for which numerical shadowing may be possible [38]
} 
We first observe that the numerical scheme (2.6) can be viewed as a discrete time dynamical system on the phase space $L^{2}$ with the notation

$$
\theta^{n+1}=F_{k}\left(\theta^{n}\right) .
$$

Thanks to the welposedness result we see that $F_{k}$ in fact maps $L^{2}$ into $H_{0, p e r}^{1}$, and $F_{k}^{2}$ maps $L^{2}$ into $H_{0, p e r}^{1} \bigcap H^{2}$ by elliptic regularity. Moreover, the discrete dynamical system is uniformly (in $k$ ) dissipative thanks to the uniform $H^{1}$ estimate $(2.20)$. Therefore, this dynamical system possesses a compact global attractor in $H^{1}$ which attracts all bounded sets in $L^{2}$. This leads to the existence of invariant measures via a classical Krylov-Bogliubov argument $[45,17]$ for the numerical scheme (the discrete dynamical system).

We recall the definition of invariant measures.

Definition 1 (Invariant measures). A Borel probability measure $\mu_{k}$ on $L^{2}$ is called an invariant measure for $F_{k}$ if

$$
\int_{L^{2}} \Phi\left(F_{k}(\theta)\right) d \mu_{k}=\int_{L^{2}} \Phi(\theta) d \mu_{k}
$$

for all bounded continuous test functional $\Phi$.

The set of all invariant measures for $F_{k}$ is denoted $\mathcal{I M}_{k}$.

We also recall that a Borel probability measure $\mu$ on $L^{2}$ is an invariant measure, or stationary statistical solution, for the infinite Prandtl number model for convection, if

1.

$$
\int_{L^{2}}\|\nabla \theta\|^{2} d \mu(\theta)<\infty
$$

2.

$\int_{L^{2}}<-R a A^{-1}(\mathbf{k} \theta) \cdot \nabla \theta-R a A^{-1}(\mathbf{k} \theta)_{3} \tau^{\prime}(z)+\Delta \theta+\tau^{\prime \prime}(z), \Phi^{\prime}(\theta)>d \mu(\theta)=0$ $(2.49)$

for any cylindrical test functional $\Phi(\theta)=\phi\left(\left(\theta, w_{1}\right), \cdots,\left(\theta, w_{m}\right)\right)$ where $\phi$ is a $C^{1}$ function on $R^{m},\left\{w_{j}, j \geq 1\right\}$ are the eigenfunctions of $\Delta$ which form an ortho-normal basis for $L^{2}$ and $w_{j} \in H_{0, p e r}^{1} \bigcap C^{2}, \forall j$, and $<,>$ denotes the 3. $H^{-1}, H_{0, p e r}^{1}$ duality.

$$
\int_{L^{2}} \int_{\Omega}\left\{|\nabla \theta|^{2}+R a\left(A^{-1}(\mathbf{k} \theta)\right)_{3} \theta \tau^{\prime}-\tau^{\prime \prime} \theta\right\} d \mathbf{x} d \mu(\theta) \leq 0
$$

The set of all stationary statistical solutions for the infinite Prandtl number model is denoted $\mathcal{I} \mathcal{M}$.

Roughly speaking, the first condition says that the invariant measures are supported on the smaller and finer space of $H^{1}$, the second condition is the differential form of the weak formulation of the invariance of the measure under the flow, and the third condition is a statistical version of the energy inequality.

Now let $\mu_{k} \in \mathcal{I} \mathcal{M}_{k}$ be s sequence of invariant measures. Thanks to the uniform estimate in $H^{1}(2.20)$, we see that the support of $\mu_{k}$ is contained in a bounded ball in $H^{1}$ independent of $k$. Therefore, thanks to the Prokhorov compactness theorem and Rellich compactness theorem $[3,33]$, the sequence $\mu_{k}$ is weakly pre-compact in 
the set of all Borel probability measures on $L^{2}$ and hence it must contain a weakly convergent subsequence (still denoted $\left\{\mu_{k}\right\}$ ) which converges to a Borel probability measure $\mu$. Our goal is to show that $\mu$ must be an invariant measure of the infinite Prandtl number model.

The first condition in the definition is easily verified since the global attractors for the discrete dynamical systems are uniformly bounded in $H^{1}$ independent of the time step $k$, and the invariant measures are supported on the global attractor [17, 51].

In order to check the second condition, i.e., the differential form of the weak formulation of invariance, we let $\Phi(\theta)=\phi\left(\left(\theta, w_{1}\right), \cdots,\left(\theta, w_{m}\right)\right)=\phi\left(y_{1}, \cdots, y_{m}\right)$ be a cylindrical test functional. Notice that

$$
\Phi^{\prime}(\theta)=\sum_{j=1}^{m} \frac{\partial}{\partial y_{j}} \phi\left(\left(\theta, w_{1}\right), \cdots,\left(\theta, w_{m}\right)\right) w_{j}
$$

hence, denoting $<,>$ the duality between $H^{-1}$ and $H_{0, p e r}^{1}$, we have

$$
\begin{aligned}
& \int_{L^{2}}<-R a A^{-1}(\mathbf{k} \theta) \cdot \nabla \theta-R a A^{-1}(\mathbf{k} \theta)_{3} \tau^{\prime}(z)+\Delta \theta+\tau^{\prime \prime}(z), \Phi^{\prime}(\theta)>d \mu(\theta) \\
= & \int_{L^{2}}<-R a A^{-1}(\mathbf{k} \theta) \cdot \nabla \theta-R a A^{-1}(\mathbf{k} \theta)_{3} \tau^{\prime}(z)+\Delta \theta+\tau^{\prime \prime}(z), \sum_{j=1}^{m} \frac{\partial \phi}{\partial y_{j}}\left(\left(\theta, w_{1}\right), \cdots,\left(\theta, w_{m}\right)\right) w_{j}>d \mu(\theta) \\
= & \int_{L^{2}} \sum_{j=1}^{m} \frac{\partial \phi}{\partial y_{j}} \int_{\Omega}\left(R a A^{-1}(\mathbf{k} \theta) \cdot \nabla w_{j} \theta-R a A^{-1}(\mathbf{k} \theta)_{3} \tau^{\prime}(z) w_{j}+\Delta w_{j} \theta+\tau^{\prime \prime}(z) w_{j}\right) d \mathbf{x} d \mu(\theta) \\
= & \lim _{k \rightarrow 0} \int_{L^{2}} \sum_{j=1}^{m} \frac{\partial \phi}{\partial y_{j}} \int_{\Omega}\left(R a A^{-1}(\mathbf{k} \theta) \cdot \nabla w_{j} \theta-R a A^{-1}(\mathbf{k} \theta)_{3} \tau^{\prime}(z) w_{j}+\Delta w_{j} \theta+\tau^{\prime \prime}(z) w_{j}\right) d \mathbf{x} d \mu_{k}(\theta) \\
= & \lim _{k \rightarrow 0} \int_{L^{2}} \sum_{j=1}^{m} \frac{\partial \phi}{\partial y_{j}} \int_{\Omega}\left(R a A^{-1}(\mathbf{k} \theta) \cdot \nabla w_{j} F_{k}(\theta)-R a A^{-1}\left(\mathbf{k} F_{k}(\theta)\right)_{3} \tau^{\prime}(z) w_{j}+\Delta w_{j} F_{k}(\theta)+\tau^{\prime \prime}(z) w_{j}\right) d \mathbf{x} d \mu_{k}(\theta) \\
= & \lim _{k \rightarrow 0} \int_{L^{2}}<-R a A^{-1}(\mathbf{k} \theta) \cdot \nabla F_{k}(\theta)-R a A^{-1}\left(\mathbf{k} F_{k}(\theta)\right)_{3} \tau^{\prime}(z)+\Delta F_{k}(\theta)+\tau^{\prime \prime}(z), \Phi^{\prime}(\theta)>d \mu_{k}(\theta) \\
= & \lim _{k \rightarrow 0} \int_{L^{2}}<\frac{F_{k}(\theta)-\theta}{k}, \Phi^{\prime}(\theta)>d \mu_{k}(\theta) \\
= & \lim _{k \rightarrow 0} \int_{L^{2}} \frac{1}{k}\left(\Phi\left(F_{k}(\theta)\right)-\Phi(\theta)\right) d \mu_{k}(\theta) \\
= & 0
\end{aligned}
$$

where we have used the boundedness and continuity of $\frac{\partial \phi}{\partial y_{j}}$ on the union of the support of $\mu_{k}$, the consistency estimate (2.24), the invariance of $\mu_{k}$ under $F_{k}$, and the following straightforward estimates valid on the union of the support of the $\mu_{k} \mathrm{~s}$ (uniformly bounded in $H_{0, p e r}^{1} \cap H^{2}$ )

$$
\begin{aligned}
\left|\int_{\Omega} A^{-1}(\mathbf{k} \theta) \cdot \nabla w_{j}\left(F_{k}(\theta)-\theta\right) d \mathbf{x}\right| & \leq c\left\|F_{k}(\theta)-\theta\right\|=\mathcal{O}(k) \rightarrow 0, \\
\left|\int_{\Omega}\left(A^{-1}\left(\mathbf{k}\left(F_{k}(\theta)-\theta\right)\right)\right)_{3} \tau^{\prime}(z) w_{j} d \mathbf{x}\right| & \leq c\left\|F_{k}(\theta)-\theta\right\|=\mathcal{O}(k) \rightarrow 0, \\
\left|\int_{\Omega} \Delta w_{j}\left(F_{k}(\theta)-\theta\right) d \mathbf{x}\right| & \leq c\left\|F_{k}(\theta)-\theta\right\|=\mathcal{O}(k) \rightarrow 0, \\
<F_{k}(\theta)-\theta, \Phi^{\prime}(\theta)> & =\Phi\left(F_{k}(\theta)\right)-\Phi(\theta)+\mathbf{o}\left(\left\|F_{k}(\theta)-\theta\right\|\right)=\Phi\left(F_{k}(\theta)\right)-\Phi(\theta)+\mathbf{o}(k),
\end{aligned}
$$


the weak convergence of $\mu_{k}$ to $\mu$, the scheme, and the invariance of $\mu_{k}$.

This proves the differential form of the weak invariance of $\mu$ under the infinite Prandtl number dynamics, i.e., no. 2.

The energy type inequality no.3 can be verified easily as well. For this purpose, we first show that any invariant measure $\mu_{k}$ of the numerical scheme (2.6) must satisfy the same energy type estimate. The desired continuous one will be the limit as the time step approaches zero.

We first show that the invariant measures for $F_{k}$ also satisfy the energy inequality. For this purpose we multiply the scheme (2.6) by $\theta^{n+1}$ and integrate over the domain, and we have

$\frac{1}{2 k}\left(\left\|\theta^{n+1}\right\|^{2}-\left\|\theta^{n}\right\|^{2}+\left\|\theta^{n+1}-\theta^{n}\right\|^{2}\right)+\left\|\nabla \theta^{n+1}\right\|^{2}+\int_{\Omega}\left(-\tau^{\prime \prime} \theta^{n+1}+R a \tau^{\prime} A^{-1}\left(\mathbf{k} \theta^{n+1}\right)_{3} \theta^{n+1}\right)=0$.

This can be re-written using the discrete dynamical system notation $F_{k}$ as

$\frac{1}{2 k}\left(\left\|F_{k}(\theta)\right\|^{2}-\|\theta\|^{2}+\left\|F_{k}(\theta)-\theta\right\|^{2}\right)+\left\|\nabla F_{k}(\theta)\right\|^{2}+\int_{\Omega}\left(-\tau^{\prime \prime} F_{k}(\theta)+R a \tau^{\prime} A^{-1}\left(\mathbf{k} F_{k}(\theta)\right)_{3} F_{k}(\theta)\right)=0$.

Integrating this identity with respect to the invariant measure $\mu_{k}$ and utilizing the invariance of $\mu_{k}$ under $F_{k}$ we have

$\int_{L^{2}} \int_{\Omega}\left(\left|\nabla F_{k}(\theta)\right|^{2}-\tau^{\prime \prime} F_{k}(\theta)+R a \tau^{\prime} A^{-1}\left(\mathbf{k} F_{k}(\theta)\right)_{3} F_{k}(\theta)\right) d \mathbf{x} d \mu_{k}(\theta)=-\frac{1}{2 k} \int_{L^{2}}\left\|F_{k}(\theta)-\theta\right\|^{2} d \mu_{k}(\theta) \leq 0$.

Utilizing the invariance of $\mu_{k}$ under $F_{k}$ again in the lower order terms we have

$$
\int_{L^{2}}\left(\left\|\nabla F_{k}(\theta)\right\|^{2}+\int_{\Omega}\left(-\tau^{\prime \prime} \theta+R a \tau^{\prime}\left(A^{-1}(\mathbf{k} \theta)\right)_{3} \theta\right) d \mathbf{x}\right) d \mu_{k}(\theta) \leq 0 .
$$

Now we recall that the support of $\mu_{k}$ is uniformly bounded in $H^{1}$ and hence, since the $w_{j}$ s are the eigenfunctions of $\Delta$ which form an orthonormal basis in $L^{2}, w_{j}$ also form a complete orthogonal system in $H_{0, p e r}^{1}$ (with the inner product between $f$ and $g$ given by $\left.\int_{\Omega} \nabla f \cdot \nabla g\right)$,

$$
\left\|\nabla F_{k}(\theta)\right\|^{2}=\lim _{m \rightarrow \infty} \sum_{j=1}^{m} \frac{\left(\nabla F_{k}(\theta), \nabla w_{j}\right)^{2}}{\left\|\nabla w_{j}\right\|^{2}}=\lim _{m \rightarrow \infty} \sum_{j=1}^{m} \frac{\left(F_{k}(\theta), \Delta w_{j}\right)^{2}}{\left\|\nabla w_{j}\right\|^{2}}
$$

Therefore

$$
\begin{aligned}
\int_{L^{2}}\left\|\nabla F_{k}(\theta)\right\|^{2} d \mu_{k}(\theta) & =\int_{L^{2}} \lim _{m \rightarrow \infty} \sum_{j=1}^{m} \frac{\left(F_{k}(\theta), \Delta w_{j}\right)^{2}}{\left\|\nabla w_{j}\right\|^{2}} d \mu_{k}(\theta) \\
& =\lim _{m \rightarrow \infty} \int_{L^{2}} \sum_{j=1}^{m} \frac{\left(F_{k}(\theta), \Delta w_{j}\right)^{2}}{\left\|\nabla w_{j}\right\|^{2}} d \mu_{k}(\theta) \\
& =\lim _{m \rightarrow \infty} \int_{L^{2}} \sum_{j=1}^{m} \frac{\left(\theta, \Delta w_{j}\right)^{2}}{\left\|\nabla w_{j}\right\|^{2}} d \mu_{k}(\theta) \\
& =\int_{L^{2}}\|\nabla \theta\|^{2} d \mu_{k}(\theta)
\end{aligned}
$$

where we have used the uniform boundedness of $\|\nabla \theta\|$ and $\left\|\nabla F_{k}(\theta)\right\|$ on the support of $\mu_{k}$, the Lebesque Dominated Convergence theorem and the invariance of $\mu_{k}$ under $F_{k}$. 
Hence we see that $\mu_{k}$ also satisfies the energy inequality, i.e.,

$$
\int_{L^{2}} \int_{\Omega}\left(|\nabla \theta|^{2}-\tau^{\prime \prime} \theta+R a \tau^{\prime}\left(A^{-1}(\mathbf{k} \theta)\right)_{3} \theta\right) d \mu_{k}(\theta) \leq 0 .
$$

Next, we take the limit as $k$ approaches zero. The last two terms in the discrete energy inequality above converge to the right limit by the very definition of weak convergence of $\mu_{k}$ to $\mu$. As for the leading order quadratic term, we have

$$
\begin{aligned}
\int\|\nabla \theta\|^{2} d \mu(\theta) & =\lim _{m \rightarrow \infty} \sum_{j=1}^{m} \int \frac{\left(\theta, \Delta w_{j}\right)^{2}}{\left\|\nabla w_{j}\right\|^{2}} d \mu(\theta) \\
& =\lim _{m \rightarrow \infty} \lim _{k \rightarrow 0} \sum_{j=1}^{m} \int \frac{\left(\theta, \Delta w_{j}\right)^{2}}{\left\|\nabla w_{j}\right\|^{2}} d \mu_{k}(\theta) \\
& \leq \liminf _{k \rightarrow 0} \sum_{j=1}^{\infty} \int \frac{\left(\theta, \Delta w_{j}\right)^{2}}{\left\|\nabla w_{j}\right\|^{2}} d \mu_{k}(\theta) \\
& =\liminf _{k \rightarrow 0} \int\|\nabla \theta\|^{2} d \mu_{k}(\theta) .
\end{aligned}
$$

This implies

$$
\begin{aligned}
& \int_{L^{2}} \int_{\Omega}\left(|\nabla \theta|^{2}-\tau^{\prime \prime} \theta+R a \tau^{\prime}\left(A^{-1}(\mathbf{k} \theta)\right)_{3} \theta\right) d \mu(\theta) \\
\leq & \liminf _{k \rightarrow 0} \int\|\nabla \theta\|^{2} d \mu_{k}(\theta)+\lim _{k \rightarrow 0} \int_{L^{2}} \int_{\Omega}\left(-\tau^{\prime \prime} \theta+R a \tau^{\prime}\left(A^{-1}(\mathbf{k} \theta)\right)_{3} \theta\right) d \mu_{k}(\theta) \\
\leq & \liminf _{k \rightarrow 0} \int_{L^{2}} \int_{\Omega}\left(|\nabla \theta|^{2}-\tau^{\prime \prime} \theta+R a \tau^{\prime}\left(A^{-1}(\mathbf{k} \theta)\right)_{3} \theta\right) d \mu_{k}(\theta)
\end{aligned}
$$

$(2.57) \leq 0$.

This completes the proof of the energy type inequality (no.3 in the definition) for the limit probability measure $\mu$. Therefore we conclude that the limit $\mu$ must be an invariant measure of the infinite Prandtl number model.

Sometimes we impose a stronger version of the statistical energy inequality in the definition of stationary statistical solutions: we require that the statistical version of the energy inequality be true on any energy shells $e_{1} \leq\|\theta\| \leq e_{2}$ instead of just one infinite shell from zero to infinity. Such kind of energy inequalities are useful in some applications in the Navier-Stokes case (see for instance [17]). They can be verified with a little bit extra work which involves approximating the finite difference by differentiation and utilizing the uniform in $\mathrm{H}^{2}$ estimates (invariant measures are supported in a bounded ball in $H^{2}$ ). We shall supply details elsewhere. Likewise it is sometimes useful to have a stronger version of the invariance of $\mu$ under the continuous in time dynamics either as a straightforward pull-back invariance or differential form of the weak invariance with a broader class of test functionals that are bounded on bounded sets of $L^{2}$, Fréchet differentiable for $\theta \in H_{0, p e r}^{1}$ with $\Phi^{\prime}(\theta) \in H_{0, p e r}^{1}$ and the derivative is continuous and bounded as a function from $H_{0, p e r}^{1}$ to $H_{0, p e r}^{1}$. It can be shown that these variations yield the same definition just as in the case of two dimensional Navier-Stokes equations [17].

Next, we turn our attention to one of the most important statistical quantities in convection: the heat transport in the vertical direction quantified by the Nusselt 
number. More specifically, we consider the limit of heat transport in the vertical direction, i.e., the Nusselt number, as the step size approaches zero. We first recall the definition of the Nusselt number.

Definition 2 (Nusselt number). For the infinite Prandtl number model, the non-dimensional averaged heat transport in the vertical direction is defined as

$$
\begin{aligned}
N u & =\sup _{\theta_{0} \in L^{2}} \limsup _{t \rightarrow \infty} \frac{1}{t L_{x} L_{y}} \int_{0}^{t} \int_{\Omega}|\nabla T(\mathbf{x}, s)|^{2} d \mathbf{x} d s \\
& =1+R a \sup _{\theta_{0} \in L^{2}} \limsup _{t \rightarrow \infty} \frac{1}{t L_{x} L_{y}} \int_{0}^{t} \int_{\Omega} A^{-1}(\mathbf{k} T(\mathbf{x}, s))_{3} T(\mathbf{x}, s) d \mathbf{x} d s \\
& =1+R a \sup _{\theta_{0} \in L^{2}} \limsup _{t \rightarrow \infty} \frac{1}{t L_{x} L_{y}} \int_{0}^{t} \int_{\Omega} A^{-1}(\mathbf{k} \theta(\mathbf{x}, s))_{3} \theta(\mathbf{x}, s) d \mathbf{x} d s
\end{aligned}
$$

Likewise, the non-dimensional averaged heat transport in the vertical direction for the discrete in time scheme (2.6) is defined as

$$
N u_{k}=1+R a \sup _{\theta_{0} \in L^{2}} \limsup _{N \rightarrow \infty} \frac{1}{N L_{x} L_{y}} \sum_{n=1}^{N} \int_{\Omega} A^{-1}\left(\mathbf{k} \theta^{n}(\mathbf{x})\right)_{3} \theta^{n}(\mathbf{x}) d \mathbf{x}
$$

It is well-known that long time averages defined through Banach (generalize) limits are spatial averages with respect to appropriate invariant measures of the underlying dynamical system $[2,17,45,46,50]$. Moreover, for a given continuous test functional $\varphi_{0}$ (in the application here $\varphi_{0}(\theta)=1+\frac{R a}{L_{x} L_{y}} \int_{\Omega} A^{-1}(\mathbf{k} \theta(\mathbf{x}))_{3} \theta(\mathbf{x}) d \mathbf{x}$ ), and a particular trajectory (initial data), there exists a particular Banach limit, $L I M_{0}$, so that $L I M_{0} \frac{1}{t} \int_{0}^{t} \varphi_{0}(\theta(s)) d s=\lim \sup \frac{1}{t} \int_{0}^{t} \varphi_{0}(\theta(s)) d s[49,50]$. Therefore, when combined with the Prokhorov's compactness theorem, we deduce the existence of an invariant measures $\mu_{k} \in \mathcal{I} M_{k}$ such that

$$
N u_{k}=1+\frac{R a}{L_{x} L_{y}} \int_{L^{2}} \int_{\Omega} A^{-1}(\mathbf{k} \theta(\mathbf{x}))_{3} \theta(\mathbf{x}) d \mathbf{x} d \mu_{k} .
$$

Hence by the weak convergence result that we just proved, we see that for any sequence of $N u_{k}$ (and hence $\mu_{k}$ ) there exists a subsequence (still denoted $N u_{k}$ and $\mu_{k}$ ), and $\mu \in \mathcal{I} \mathcal{M}$ such that

$$
\begin{aligned}
\lim _{k \rightarrow 0} N u_{k} & =1+\frac{R a}{L_{x} L_{y}} \lim _{k \rightarrow 0} \int_{L^{2}} \int_{\Omega} A^{-1}(\mathbf{k} \theta(\mathbf{x}))_{3} \theta(\mathbf{x}) d \mathbf{x} d \mu_{k} \\
& =1+\frac{R a}{L_{x} L_{y}} \int_{L^{2}} \int_{\Omega} A^{-1}(\mathbf{k} \theta(\mathbf{x}))_{3} \theta(\mathbf{x}) d \mathbf{x} d \mu \\
& \leq 1+\frac{R a}{L_{x} L_{y}} \sup _{\nu \in \mathcal{I} \mathcal{M}} \int_{L^{2}} \int_{\Omega} A^{-1}(\mathbf{k} \theta(\mathbf{x}))_{3} \theta(\mathbf{x}) d \mathbf{x} d \nu \\
& =1+\frac{R a}{L_{x} L_{y}} \sup _{\theta_{0} \in L^{2}} \lim _{t \rightarrow \infty} \frac{1}{t L_{x} L_{y}} \int_{0}^{t} \int_{\Omega} A^{-1}(\mathbf{k} \theta(\mathbf{x}, s))_{3} \theta(\mathbf{x}, s) d \mathbf{x} d s
\end{aligned}
$$

where we have used the weak convergence of $\mu_{k}$ to $\mu$, the compactness of the set of all invariant measures due to Prokhorov's theorem and the a priori estimates, the fact that extremal points of the set of invariant measures are ergodic (in the sense that phase space spatial average and time average are the same) [2, 46, 49]. 
To summarize, we have proved the following main result.

THEOREM 1 (Convergence of stationary statistical properties). Let $\mu_{k}$ be an arbitrary invariant measure of the numerical scheme (2.6) with time step $k$, i.e. $\mu_{k} \in \mathcal{I M}_{k}$, and let $N u_{k}$ be the Nusselt number characterizing the heat transport in the vertical direction for the scheme with time step $k$ defined in (2.59). Then each subsequence of $\mu_{k}$ must contain a subsubsequence (still denoted $\left\{\mu_{k}\right\}$ ) and an invariant measure $\mu$ of the infinite Prandtl number model so that $\mu_{k}$ weakly converges to $\mu$, i.e.,

$$
\mu_{k} \rightarrow \mu, k \rightarrow 0 .
$$

Moreover, the Nusselt number converges in an upper semi-continuous fashion in the sense that

$$
\limsup _{k \rightarrow 0} N u_{k} \leq N u .
$$

In particular, this implies that the convergent numerical schemes will not overestimate the Nusselt number asymptotically.

Notice that our asymptotic lower bound on the Nusselt number for the infinite Prandtl number model nicely complements the rigorous upper bound for the Nusselt number using variational approach proposed by Constantin and Doering [7, 8].

3. Conclusions and Remarks. Our main result clearly demonstrated the usefulness of the uniformly dissipative scheme that we proposed in terms of approximating stationary statistical properties of the infinite Prandtl number model for convection since the stationary statistical properties of the scheme converge to those of the continuous time model. To the best of our knowledge, this is the first rigorous result proving convergence of stationary statistical properties of numerical schemes to those of the continuous in time dynamical system under approximation. Our result may be viewed as a partial generalization of Lax's equivalence theorem in the sense that consistency and long time stability (uniform dissipativity) implies convergence of stationary statistical properties. We would like to emphasize that the methodology here can be applied to much more general dissipative systems (with chaotic behavior for relevance) although we have treated the infinite Prandtl number model only [51]. We hope that our work will stimulate further study on numerical schemes for approximating statistical properties of dissipative dynamical systems.

The convergence of the stationary statistical properties rely on the uniform bound in a space which is compactly embedded in the phase space (it is $H_{0, p e r}^{1}$ in the infinite Prandtl number case which is compactly imbedded in the phase space $L^{2}$ by Rellich's theorem). Simply having a uniform bound in the phase space may not imply the convergence of the statistical properties (we could construct schemes that possess absorbing ball in $L^{2}$ but not in $H^{1}$ ). Therefore, we would rather use the uniformly dissipative terminology instead of the global in time stability used by many other authors which could mean uniform boundedness in the phase space only.

The convergence that we derived here is actually semi-convergence since different subsequences may converge to different invariant measures of the continuous in time dynamical system. There is no convergence rate either. This is perhaps the generic picture in the sense that the result here is nearly optimal without additional assumption on the continuous dynamical system. One very useful physical assumption is mixing of the continuous system. Indeed, if we assume that the continuous system is 
exponentially mixing with a rate of $r$ [46], i.e., there is a physically relevant invariant measure $\mu$ so that

$$
\left|\frac{1}{t} \int_{0}^{t} \Phi(\theta(\mathbf{x}, s)) d s-\int \Phi(\theta(\mathbf{x})) d \mu\right| \leq c \exp (-r t)
$$

for all appropriate test functionals $\Phi(\theta)$ and almost all trajectories, then approximating a specific statistical quantity $\int \Phi(\theta(\mathbf{x})) d \mu$ becomes a finite time integration problem. Indeed, suppose the given tolerance level is $2 \epsilon$, we first fix a time $t$ so that $c \exp (-r t) \leq \epsilon$ (the time $t$ is usually large for small mixing rate $r$ ). We then adjust our mesh size (small time step or mesh size) so that

$$
\left|\frac{1}{t} \int_{0}^{t} \Phi(\theta(\mathbf{x}, s)) d s-\frac{1}{N} \sum_{n=1}^{N} \Phi\left(\theta^{n}\right)\right| \leq \epsilon
$$

where $N k=t$ with $k$ being the time step. Hence the infinite time approximation of stationary statistical property becomes the problem of approximation on finite time interval $[0, t]$ for appropriate numerical schemes (say uniformly dissipative). This motives us to work on higher order schemes so that the integration on $[0, t]$ can be calculated quickly.

Of course we do not have exponential mixing for generic dissipative complex/chaotic dynamical systems. One way to circumvent this difficulty is by considering noisy systems since our environment is intrinsically noisy. Exponential mixing can be verified for many dissipative systems with appropriate additive white noise [11, 13, 35, 32, 14]. Hence there is a strong incentive to generalize the notion of uniformly dissipative schemes to approximations of continuous in time stochastic dynamical systems (both SDE and SPDE, see [35] for the case of SDE and fully implicit approach). We will report results in this direction somewhere else.

The scheme that we presented here is not the only scheme that is able to capture stationary statistical properties of the underlying continuous system. The fully implicit backward Euler scheme is a uniformly dissipative scheme as one can readily verify. However, the backward Euler is nonlinear in the unknown and hence the computational cost at each time step is expected to be higher. There are other linear implicit uniformly dissipative schemes. For instance one may check that the following family of schemes is uniformly dissipative for $\lambda \in[0,1]$,

$\frac{\theta^{n+1}-\theta^{n}}{k}+R a A^{-1}\left(\mathbf{k} \theta^{n}\right) \cdot \nabla \theta^{n+1}+R a\left(A^{-1}\left(\mathbf{k}\left(\lambda \theta^{n+1}+(1-\lambda) \theta^{n}\right)\right)\right)_{3} \tau^{\prime}(z)=\Delta \theta^{n+1}+\tau^{\prime \prime}(z)$.

However, it seems that this family of scheme is uniformly dissipative under small time step restriction $k \leq \frac{1}{R a^{2}}$. This kind of restriction may be expected since the linearly unstable modes grow as the Rayleigh number grows and hence the time step should reflect this through CFL condition. On the other hand, we observe that for the case of $\lambda=0$, the scheme is the same as

$$
\frac{T^{n+1}-T^{n}}{k}+R a A^{-1}\left(\mathbf{k} T^{n}\right) \cdot \nabla T^{n+1}=\Delta T^{n+1}
$$

whose inviscid part is stable (satisfies maximum principle). Hence if the viscous scheme is unstable, the viscous term plays a destabilizing role. We also notice that the case with $\lambda=0$ corresponds to discretization in time first followed by translation as we mentioned in section 2.2 . 
It is also worthwhile to point out that at the time discretization only stage we should anticipate implicit scheme due to the CFL condition.

An issue that we have not addressed here is spatial discretization. Since we have utilized the background temperature profile $\tau$ in our uniform dissipativity argument, it is expected that we need to resolve small scales within the background profile. Similar issue for the Navier-Stokes was investigated earlier [9]. We will report the details somewhere else.

Another issue that we have not addressed here is the behavior of the global attractors. We fully anticipate an upper semi-continuity result. The proof is a modification of the classical one $[42,37,21]$ since we will not have uniform in time convergence of trajectories on finite time interval. In fact, we will have uniform in time convergence after a transitional period of time due to the fact that the points on the global attractors of the scheme may not satisfy high order compatibility condition for the infinite Prandtl number model and hence the solution may have an initial transitional layer (see [22] for the case of two dimensional Navier-Stokes equations). We will report this elsewhere as well.

Acknowledgement. This work is supported in part by grants from NSF. Part of the work was done while XW was a visitor at Courant Institute at New York University, and at the Institute for Applied Mathematics at the University of Bonn. We also acknowledge helpful conversations with Brian Ewald and Andy Majda.

\section{REFERENCES}

[1] Amati, G., Koal, K., Massaioli, F., Sreenivasan, K.R., and Verzicco, R.; Turbulent thermal convection at large Rayleigh numbers for a Boussinesq fluid of constant Prandtl number, Physics of Fluids, 17 (12), 2005.

[2] Bekka, M. B. and Mayer, M.; Ergodic theory and topological dynamics of group actions on homogeneous spaces, Cambridge, U.K. ; New York : Cambridge University Press, 2000. ( London Mathematical Society lecture note series ; 269)

[3] Billingsley, P.; Weak convergence of measures: applications in probability. SIAM, Philadelphia, 1971.

[4] Bodenschatz, E., Pesch, W. and Ahlers, G.; Recent developments in Rayleigh-Bénard convection. Annual review of fluid mechanics, Vol. 32, 709-778, 2000.

[5] Calzavarini, E., Doering, C.R., Gibbon, J.D., Lohse, D., Tanabe, A. and Toschi, F.; Exponentially growing solutions in homogeneous Rayleigh-Bénard convection, Phys. Rev. E., 73(3), 035301(4), 2006.

[6] Chandrasekhar, S.; Hydrodynamic and hydro-magnetic stability. Oxford, Clarendon Press, 1961.

[7] Constantin, P. and Doering, C.R.; Heat transfer in convective turbulence, Nonlinearity, 9, (1996), 1049-1060.

[8] Constantin, P. and Doering, C.R.; Infinite Prandtl number convection, J. Stat. Phys. 94 (1999), no. 1-2, 159-172.

[9] Cheng, W. and Wang, X.; A discrete Kato type theorem on inviscid limit of Navier-Stokes flows, J. МАтн. PHYs., vol.48, no.6, 065303 (2007).

[10] Cheng, W. and Wang, X.; A uniformly dissipative scheme for stationary statistical properties of the infinite Prandtl number model for convection, Applied Mathematics LetTers, accepted 2007, in press. doi:10.1016/j.aml.2007.07.036

[11] Da Prato, G. and Zabczyk, J.; Ergodicity for infinite dimensional systems. Cambridge ; New York : Cambridge University Press, 1996.

[12] Doering, C.R., Otto, F. and Reznikoff, M.G.; Bounds on vertical heat transport for infinite Prandtl number Rayleigh-Bénard convection, J. Fluid Mech. (2006), vol. 560, 229-241.

[13] E, Weinan; Stochastic hydrodynamics. Current developments in mathematics, 2000, 109-147, Int. Press, Somerville, MA, 2001

[14] E, Weinan and Li, D.; The Andersen thermostat in molecular dynamics, preprint, 2007.

[15] Foias, C., Jolly, M., Kevrekidis, I.G. and Titi,E.S.; Dissipativity of numerical schemes, Nonlinearity 4 (1991), 591-613 
[16] Foias, C., Jolly, M., Kevrekidis, I.G. and Titi,E.S.; On some dissipative fully discrete nonlinear Galerkin schemes for the Kuramoto-ivashinsky equation, Phys. Lett. A 186 (1994), 87-96.

[17] Foias, C., Manley, O., Rosa, R. and Temam, R.; Navier-Stokes equations and turbulence. Encyclopedia of Mathematics and its Applications, 83. Cambridge University Press, Cambridge, 2001.

[18] Getling, A. V.; Rayleigh-Bénard convection. Structures and dynamics. Advanced Series in Nonlinear Dynamics, 11. World Scientific Publishing Co., Inc., River Edge, NJ, 1998.

[19] Geveci, T.; On the convergence of a time discretization scheme for the Navier-Stokes equations, Math. Comp., 53 (1989), 43-53.

[20] Grossmann, S. and Lohse, D.; Scaling in thermal convection: a unifying theory, J. Fluid Mech., vol. 407, 2000, 27-56.

[21] Hale, J. K.; Asymptotic behavior of dissipative systems, Providence, R.I. : American Mathematical Society, 1988.

[22] Heywood, J. G. and Rannacher, R.; Finite element approximation of the nonstationary NavierStokes problem. I. Regularity of solutions and second order error estimates for spatial discretization. SIAM J. Numer. Anal. 19 (1982), no. 2, 275-311.

[23] Heywood, J. G. and Rannacher, R.; Finite element approximation of the nonstationary NavierStokes problem. II. Stability of solutions and error estimates uniform in time. SIAM J. Numer. Anal. 23 (1986), no. 4, 750-777.

[24] Heywood, J. G. and Rannacher, R.; Finite-Element Approximation of the Nonstationary Navier-Stokes Problem Part IV: Error Analysis for Second-Order Time Discretization, SIAM J. Numer. Anal., Vol. 27, No. 2 (Apr., 1990), 353-384

[25] Hill, A.T. and Süli, E.; Approximation of the global attractor for the incompressible NavierStokes equations, IMA Jour. Numer. Anal., 20 (2000), 663-667.

[26] Howard, L.; Heat transport by turbulent convection, J. Fluid Mech. 17, 1963, pp.405-432.

[27] Ierley, G.R., Kerswell, R.R. and Plasting, S.C.; Infinite-Prandtl-number convection. Part 2. A singular limit of upper bound theory, J. Fluid Mech. (2006), vol. 560, 157-227.

[28] Jolly, M., Kevrekidis, I., and Titi, E.S.; Preserving dissipation in approximate inertial forms for the Kuramoto-Sivashinsky equation, Journal of Dynamics and Differential Equations 3 (1991), 179-197.

[29] Ju, N.; On the global stability of a temporal discretization scheme for the Navier-Stokes equations, IMA J. Numer. Anal., 22 (2002), 577-597.

[30] Kadanoff, L.P.; Turbulent heat flow: structures and scaling, Physics Today, 54, no. 8, pp.34-39, 2001.

[31] Larsson, S.; The long-time behavior of finite-element approximations of solutions to semilinear parabolic problems. SIAM J. Numer. Anal. 26 (1989), no. 2, 348-365.

[32] Lasota, A. and Mackey, M.C.; Chaos, fractals, and noise : stochastic aspects of dynamics, 2nd ed. , New York : Springer-Verlag, 1994.

[33] Lax, P.D.; Functional Analysis, New York : Wiley, 2002.

[34] Majda, A.J. and Wang, X.; Nonlinear Dynamics and Statistical Theory for Basic Geophysical Flows, CAmbridge University Press, Cambridge, England, 2006.

[35] Mattingly, J. C., Stuart, A. M. and Higham, D. J.; Ergodicity for SDEs and approximations: locally Lipschitz vector fields and degenerate noise. Stochastic Process. Appl. 101 (2002), no. 2, 185-232.

[36] Monin, A.S. and Yaglom, A.M.; Statistical fluid mechanics; mechanics of turbulence, English ed. updated, augmented and rev. by the authors. MIT Press, Cambridge, Mass., 1975.

[37] Raugel, G.; Global attractors in partial differential equations. Handbook of dynamical systems, Vol. 2, 885-982, North-Holland, Amsterdam, 2002.

[38] Sauer, T. and Yorke, J.A.; Rigorous verification of trajectories for the computer simulation of dynamical systems, NONLINEARITY 4 (1991), 961-979.

[39] Shen, J.; Long time stabilities and convergences for the fully discrete nonlinear Galerkin methods, Appl. Anal., 38 (1990), 201-229.

[40] Siggia, E.; High Rayleigh number convection. Annual review of fluid mechanics, Vol. 26, 137168, Annual Reviews, Palo Alto, CA, 1994.

[41] Temam, R.M.; Navier-Stokes Equations and Nonlinear Functional Analysis, 2nd edition, CBMS-SIAM, SIAM, 1995.

[42] Temam, R.M.; Infinite Dimensional Dynamical Systems in Mechanics and Physics, 2nd ed, Springer-Verlag, New York, 1997.

[43] Tone, F. and Wirosoetisno, D.; On the long-time stability of the implicit Euler scheme for the two-dimensional Navier-Stokes equations. SIAM J. Numer. Anal. 44 (2006), no. 1, 29-40

[44] Tritton, D.J.; Physical Fluid Dynamics, Oxford Science Publishing, 1988.

[45] Vishik, M.I. and Fursikov, A.V.; Mathematical Problems of Statistical Hydromechanics, Kluwer 
Acad. Publishers, Dordrecht/Boston/London, 1988.

[46] Walters, P.; An introduction to ergodic theory. Springer-Verlag, New York, 2000.

[47] Wang, X.; Infinite Prandtl Number Limit of Rayleigh-Bénard Convection, Comm. Pure AND ApPl. Math., vol 57, issue 10, (2004), 1265-1282.

[48] Wang, X.; Asymptotic behavior of global attractors to the Boussinesq system for RayleighBénard convection at large Prandtl number, Comm. Pure and Appl. Math., vol, 60, no. 9, 1293-1381, 2007.

[49] Wang, X.; Stationary statistical properties of Rayleigh-Bénard convection at large Prandtl number, COMm. PURe AND ApPl. Math., vol, 61, no. 6, 0789-0815, 2008.

[50] Wang, X.; Lecture Notes on Elementary Statistical Theories with Applications to Fluid Systems, 2007 Shanghai Mathematics Summer School in Fudan University. To be published by High Education Press.

[51] Wang, X.; 2008, Temporal approximations of stationary statistical properties of dissipative systems, submitted to CPAM. 